\title{
Instability of time-dependent wind-driven ocean gyres
}

\author{
Paul C. F. van der Vaart \\ Institute for Marine and Atmospheric Research Utrecht, Department of Physics and Astronomy, \\ Utrecht University, Utrecht, the Netherlands \\ Henk M. Schuttelaars \\ Faculty of Civil Engineering and Geosciences, Delft University of Technology, Delft, the Netherlands \\ Daniel Calvete \\ Department Fisica Aplicada, UPC, Barcelona, Spain
}

Henk A. Dijkstra ${ }^{a}$

Institute for Marine and Atmospheric Research Utrecht, Department of Physics and Astronomy, Utrecht University, Utrecht, the Netherlands

(Received 22 February 2002; accepted 10 July 2002; published 5 September 2002)

\begin{abstract}
The wind-driven ocean circulation at midlatitudes is susceptible to several types of instabilities. One of the simplest models of these flows is the quasigeostrophic barotropic potential vorticity equation in an idealized ocean basin. In this model, the route to complex spatio/temporal flows is through successive bifurcations. The aim of this study is to describe the physics of the destabilization process of a periodic wind-driven flow associated with a secondary bifurcation. Although bifurcation theory has proven to be a valuable tool to determine the physical mechanisms of destabilization of fluid flows, the analysis of the stability of time-dependent (for example, periodic) flows, using this methodology, is computationally unpractical, due to the large number of degrees-of-freedom involved. The approach followed here is to construct a low-order model using numerical Galerkin projection of the full model equations onto the dynamically active eigenmodes. The resulting reduced model is shown to capture the local dynamics of the full model. The physical mechanism of the destabilization of the periodic wind-driven flow is deduced from the reduced model. While there are several stabilizing processes, notably rectification, the destabilization occurs due to time-dependent increase of the background horizontal shear in the flow. (C) 2002 American Institute of Physics. [DOI: 10.1063/1.1503804]
\end{abstract}

\section{INTRODUCTION}

The midlatitude, mainly wind-driven, surface circulation in the ocean is characterized by gyre-type circulations which are closed by strong western boundary currents. For example, the Gulf Stream is the western boundary current in the North-Atlantic and is part of a system of two gyres, the subtropical and subpolar gyre. The intensification of the flows at the western boundaries of ocean basins is a classical problem in dynamical oceanography, of which the basics are understood for a long time. ${ }^{1,2}$ The internal variability of these boundary currents has recently been recognized as one possible source of low-frequency variability (i.e., on time scales of years to decades) in the climate system. ${ }^{3}$

Western boundary currents are highly nonlinear and hence instabilities of such flows occur on several space and time scales. Eddy-resolving ocean models are a frequently used tool to study the internal temporal variability of the midlatitude wind-driven ocean circulation. ${ }^{4}$ With the possibility to perform numerical simulations on long time intervals and at high horizontal resolution, this low-frequency variability has been extensively studied and was shown to be associated with the occurrence of low- and high-kinetic en-

a)Electronic mail: dijkstra@phys.uu.nl ergy states. ${ }^{5,6}$ The dynamical and physical origin of this type of variability is a subject of intense study. ${ }^{7-9}$

Much progress has been made through bifurcation studies which uncovered the structure of steady states and largescale instabilities in idealized models. ${ }^{10-12}$ Usually, only the first couple of Hopf bifurcations are determined and the periodic solution arising from the first Hopf bifurcation is followed. It was shown that the first Hopf bifurcations introduce intermonthly and interannual variability into the barotropic flows. Already this information has provided an interpretational framework for high resolution simulations ${ }^{6}$ in the relatively high (lateral) friction range. Recent work on a more complete structure of Hopf bifurcations for a twolayer shallow-water model, combined with the computation of many trajectories, has indicated that the low-frequency variability associated with the high- and low-energy states can be described within a finite-dimensional framework. Only a small number of dynamical modes seems to be involved to cause this type of behavior. ${ }^{9}$

However, what is still lacking is the precise dynamical behavior far from critical conditions, the latter defined by the location of the first Hopf bifurcation. Transient flow computations are inadequate in resolving the full bifurcation structure because unstable steady states and periodic orbits cannot be determined. This is a more general problem in hydrody- 
namic stability theory. ${ }^{13,14}$ For systems of partial differential equations, computation of periodic orbits in parameter space and their subsequent stability analysis (Floquet theory) is not yet practical, because of the large number of degrees of freedom involved. Hence, the reduction of the governing equations of motion to systems of ordinary differential equations provides a way to make progress in understanding the route to complexity and corresponding physical mechanisms of transition within these flows.

There are many examples in the fluid physics literature of model reduction which can be summarized as (Galerkintype) projections of the full equations on a set of basis functions. ${ }^{15}$ In many cases, the reduced model consists of a set of amplitude equations (e.g., Refs. 16 and 17). For the basis functions, one can take (i) eigenfunctions of a particular linear operator, ${ }^{18}$ (ii) statistically determined basis functions such as empirical orthogonal functions or (iii) orthogonal polynomials (as in spectral methods). Many problems are known with these types of low-order models and the main frustration is the noncorrespondence in dynamical behavior between the reduced model and the full model when the order of truncation of the former is changed. An enormously rich behavior may be found in many reduced models, which sometimes has fascinated researchers so much that critically examining the relation between the full and reduced models is omitted. An example hereof is the famous Lorenz model, ${ }^{19}$ where the dynamics bear little resemblance to that of the underlying full model of Rayleigh-Bénard convection. ${ }^{20}$

With these problems in mind, and accepting that it may be very difficult to find a low-order model which can capture the dynamics of a full model over a reasonable range in parameter space, our approach here is less ambitious. The main aim of this paper is to construct a low-order model that allows one to determine the physical mechanism of the destabilization of a limit cycle, which arises through a Hopf bifurcation of the steady wind-driven barotropic quasigeostrophic $(\mathrm{QG})$ double gyre flow. ${ }^{12}$ We tackle this problem by using (i) continuation techniques to determine steady states and Hopf bifurcations within the full model, (ii) transient flow computations at several locations in parameter space, and (iii) locally reduced models to determine the stability of the periodic orbits by means of Floquet analysis. In the latter models, nonorthogonal basis functions are used which have an immediate physical interpretation. Representation issues of the low-order model are evaluated by comparing the results directly with those of the full model. The limit cycle destabilizes through a Neimark-Sacker bifurcation and the physics of the destabilization process is described with help of the reduced model.

The setup of the paper is as follows. In Sec. II the QG model will be described, followed by a description of the numerical continuation techniques. The general derivation of the low-order model is then presented in Sec. II C. The description of the dynamics of the full QG model in Sec. III is followed by the construction and analysis of the locally reduced model in Sec. IV. The physical mechanism associated with the Neimark-Sacker bifurcation is presented in Sec. V. A summary and discussion of the main results conclude this paper.

\section{METHODOLOGY}

We consider ocean flows with constant density $(\rho)$ in a rectangular basin of horizontal dimensions $L$ and $B$ and with constant depth $D$, which is situated on a midlatitude $\beta$ plane with Coriolis parameter $f=f_{0}+\beta_{0} y$. The ocean circulation in the basin is forced at the surface through a wind-stress vector $\tau=\tau_{0}\left(\tau^{x}(x, y), \tau^{y}(x, y)\right)$.

\section{A. The quasigeostrophic model}

In a quasigeostrophic approximation, the flow can be modelled by the well-known barotropic potential vorticity equation (Pedlosky, 1987) for the geostrophic stream function $\psi$. This equation is nondimensionalized by using horizontal and vertical length scales $L$ and $D$, a characteristic horizontal velocity scale $U$, a time scale $L / U$, and the maximum amplitude of the wind stress $\tau_{0}$. With introduction of the horizontal velocities $u$ and $v$ and the relative vorticity $\zeta$, the dimensionless model can be written as

$$
\begin{gathered}
{\left[\frac{\partial}{\partial t}+u \frac{\partial}{\partial x}+v \frac{\partial}{\partial y}\right](\zeta-F \psi+\beta y)} \\
=\frac{1}{R} \nabla^{2} \zeta+\alpha_{\tau}\left(\frac{\partial \tau^{y}}{\partial x}-\frac{\partial \tau^{x}}{\partial y}\right), \\
\zeta=\nabla^{2} \psi, \\
u=-\frac{\partial \psi}{\partial y}, \quad v=\frac{\partial \psi}{\partial x} .
\end{gathered}
$$

These equations have been used many times to study phenomena in geophysical flows (e.g., Refs. 21 and 22). On the lateral zonal boundaries $x=0,1$, no-slip conditions are prescribed whereas at the meridional boundaries $(y=0, A)$, we apply slip conditions

$$
\begin{aligned}
& x=0,1, \quad \psi=0, \quad \frac{\partial \psi}{\partial x}=0, \\
& y=0, A, \quad \psi=0, \quad \zeta=0 .
\end{aligned}
$$

Besides the aspect ratio $A=B / L$, several other parameters appear, i.e., the Reynolds number $R$, the strength of the planetary vorticity gradient $\beta$, the wind-stress forcing strength $\alpha_{\tau}$ and the homogeneous Froude number $F$. The parameters are defined as

$R=\frac{U L}{A_{H}}, \quad \beta=\frac{\beta_{0} L^{2}}{U}, \quad \alpha_{\tau}=\frac{\tau_{0} L}{\rho D U^{2}}, \quad F=\frac{f_{0}^{2} L^{2}}{g D}$,

where $A_{H}$ is the lateral friction coefficient and $g$ the gravity acceleration.

The characteristic velocity $U$ is chosen such that $\alpha_{\tau}$ $=\beta$ to give Sverdrup flow over the main part of the basin. The wind stress considered is

$\tau^{x}(y)=-\frac{1}{2 \pi}\left[(1-a) \cos \left(\frac{2 \pi y}{A}\right)-a \sin \left(\frac{2 \pi y}{A}\right)\right], \quad \tau^{y}=0$,

where the parameter $a$ controls the asymmetry of the windstress field with respect to the midaxis of the basin. The unforced system of equations and boundary conditions ad- 
mits a reflection symmetry through the midaxis $(y=A / 2)$ of the basin. This reflection $\mathcal{R}$ has a representation

$$
\mathcal{R}(\psi(x, A-y))=-\psi(x, y) .
$$

For $a=0$, the applied wind stress admits a similar symmetry and this puts constraints on the bifurcations, i.e., symmetry breaking bifurcations will be of pitchfork type. ${ }^{23}$

\section{B. Steady states and their stability}

The equations are discretized using central differences on an equidistant $N \times M$ grid. After discretization, a system of nonlinear differential equations with algebraic constraints results, which can be written as

$$
\mathcal{M} \frac{d \phi}{d t}+\mathbf{F}(\phi, \lambda)=\mathbf{0} .
$$

Here $\phi$ is the $d$-dimensional state vector $(d=2 \times N \times M)$, consisting of the unknowns at each grid point, $\lambda$ can be any of the parameters $(R, \beta, A, F, a), \mathbf{F}$ is a nonlinear mapping from $R^{d} \times R \rightarrow R^{d}$ and the mass-matrix $\mathcal{M}$ is a linear operator.

Stationary solutions $\boldsymbol{\Phi}$ satisfy the equation

$$
\mathbf{F}(\boldsymbol{\Phi}, \lambda)=0
$$

which is a system of $d$ nonlinear algebraic equations. To compute a branch of stationary solutions in one of the control parameters, a pseudoarclength continuation method ${ }^{24}$ is used. The branches of stationary solutions $(\boldsymbol{\Phi}(s), \lambda(s))$ are parametrized by an arclength parameter $s$. Since this introduces an extra unknown, an additional equation is needed and the tangent is normalized along the branch, i.e.,

$$
\dot{\boldsymbol{\Phi}}_{0}^{T}\left(\boldsymbol{\Phi}-\boldsymbol{\Phi}_{0}\right)+\dot{\lambda}_{0}^{T}\left(\lambda-\lambda_{0}\right)-\Delta s=0,
$$

where $\Delta s$ is the step length and a dot indicates differentiation to $s .\left(\Phi_{0}, \lambda_{0}\right)$ indicates the solution at the previous step. The Newton-Raphson method is used to converge to the branch of stationary solutions. This method finds isolated steady solutions, regardless of their stability. The linear systems are solved with a preconditioned conjugate gradient method. ${ }^{25}$

When a steady state has been determined, the linear stability of the solution is considered and transitions that mark qualitative changes such as transitions to multiple equilibria (pitchfork bifurcations or limit points) or periodic behavior (Hopf bifurcations) can be detected. The linear stability analysis amounts to solving a generalized eigenvalue problem of the form

$$
\gamma \mathcal{J} \tilde{\phi}=\kappa \mathcal{M} \tilde{\phi}
$$

where $\mathcal{J}$ is the Jacobian matrix of (7). The matrices $\mathcal{J}$ and $\mathcal{M}$ are in general nonsymmetric matrices, with $\mathcal{M}$ being singular, and $\gamma$ and $\kappa$ complex numbers. The problem (9) is solved using the QZ method. ${ }^{26}$ In case $\gamma \neq 0$ in (9), we will use the notation $\sigma=\sigma_{r}+i \sigma_{i}=\kappa / \gamma$ to indicate the eigenvalue. Hopf bifurcation points are detected by monitoring the eigenvalues along a branch of steady solutions. If $\sigma_{r}$ changes sign while $\sigma_{i} \neq 0$ at some value of the control parameter $\lambda$, a secant method is used to determine the value of $\lambda$ at Hopf bifurcation where $\sigma_{r}=0$.

\section{Locally reduced models}

The discretized system of equations, as described above, will be referred to below as the full model. Suppose that at some value of the control parameter, say at $R=R_{H}$, a Hopf bifurcation has been detected within this full model using the methods described above. From general theory, it is known that locally a limit cycle exists which may be stable (supercritical Hopf) or unstable (subcritical Hopf). ${ }^{27}$ When the dimension of the discretized system $d$ is very large, it becomes computationally expensive to determine these limit cycles directly from the (discretized) partial differential equations, although general methodology exists. ${ }^{28}$ The idea pursued here is to construct (numerically) a low-order amplitude equation model, locally near the Hopf bifurcation, which is capable to represent the dynamics of the full model beyond the weakly nonlinear regime.

The set of discretized nonlinear differential equations (6) is rewritten in the general form

$$
\mathcal{M} \frac{d \phi}{d t}+\mathcal{L} \phi+\mathcal{N}(\phi, \phi)=\mathcal{F}
$$

where the nonlinear operator $\mathbf{F}$ has been replaced by

$$
\mathbf{F}=\mathcal{L} \phi+\mathcal{N}(\phi, \phi)-\mathcal{F}
$$

to explicitly show the linear operator $\mathcal{L}$, the nonlinear operator $\mathcal{N}$ and the forcing $\mathcal{F}$. Let $\boldsymbol{\Phi}$ be the solution to the steady problem, i.e.,

$$
\mathcal{L} \boldsymbol{\Phi}+\mathcal{N}(\boldsymbol{\Phi}, \boldsymbol{\Phi})=\mathcal{F} .
$$

The solution to (10) is now decomposed into this steady state and a time-dependent part,

$$
\phi=\boldsymbol{\Phi}+\widetilde{\phi}
$$

After substitution into (10), the linearized flow $\widetilde{\phi}$ is governed by

$$
\mathcal{M} \frac{d \tilde{\phi}}{d t}+\mathcal{J} \tilde{\phi}=0,
$$

where the total Jacobian $\mathcal{J}$ is defined as

$$
\mathcal{J} \equiv \mathcal{L}+\mathcal{N}(\boldsymbol{\Phi}, \cdot)+\mathcal{N}(\cdot, \boldsymbol{\Phi}) .
$$

The linear operator $\mathcal{J}$ has an eigenvector decomposition,

$$
\mathbf{L}^{H} \mathcal{J} \mathbf{R}=\Sigma, \quad \mathbf{L}^{H} \mathcal{M} \mathbf{R}=\mathbf{I} .
$$

Here $\mathbf{R}$ and $\mathbf{L}$ denote the right- and left-hand eigenspaces of the linear operator $\mathcal{J}, \mathbf{I}$ is the identity and the diagonal matrix $\Sigma$ contains the corresponding eigenvalues, i.e.,

$$
\begin{aligned}
& \mathbf{R}=\left(\begin{array}{llll}
\mathbf{r}_{1} & \mathbf{r}_{2} & \cdots & \mathbf{r}_{r}
\end{array}\right), \\
& \mathbf{L}=\left(\begin{array}{llll}
\mathbf{l}_{1} & \mathbf{l}_{2} & \cdots & \mathbf{l}_{r}
\end{array}\right), \\
& \Sigma=\operatorname{diag}\left(\begin{array}{llll}
\sigma_{1} & \sigma_{2} & \cdots & \sigma_{r}
\end{array}\right),
\end{aligned}
$$

where $r=\operatorname{rank}(\Sigma)=(M-2) \times(N-2)<d$; the latter inequality is due to the singular nature of $\mathcal{M}$. Relation (14) states that $\mathbf{L}$ and $\mathbf{R}$ are a bi-orthogonal set of eigenvectors (i.e., $\mathbf{l}_{j} M \mathbf{r}_{k}=\delta_{j k}$ ) and this property will be used in the Galerkin projection below. 
TABLE I. Dimensional and nondimensional parameter values.

\begin{tabular}{lc}
\hline \hline$L=10^{6} \mathrm{~m}$ & $F=0$ \\
$\beta_{0}=1.6 \times 10^{-11} \mathrm{~m}^{-1} \mathrm{~s}^{-1}$ & $A=1$ \\
$f_{0}=1.0 \times 10^{-4} \mathrm{~s}^{-1}$ & $\beta=10^{3}$ \\
$\rho=1.002 \times 10^{3} \mathrm{~kg} \mathrm{~m}^{-3}$ & $\alpha_{\tau}=10^{3}$ \\
$\tau_{0}=0.128 \mathrm{~kg} \mathrm{~m}^{-1} \mathrm{~s}^{-2}$ & $a=0.2$ \\
$D=500 \mathrm{~m}$ & \\
$U=1.6 \times 10^{-2} \mathrm{~m} \mathrm{~s}^{-1}$ & \\
\hline
\end{tabular}

With the use of this eigenbasis, the perturbation $\widetilde{\phi}$ is expanded in $n$ right-hand eigenvectors,

$$
\widetilde{\phi}=\mathbf{R}_{n} \mathbf{a}=\sum_{j=1}^{n} \mathbf{r}_{j} a_{j}(t) .
$$

The matrix $\mathbf{R}_{n}$ denotes the $n$-dimensional subspace of $\mathbf{R}$ of suitably chosen right-hand vectors, and $\mathbf{L}_{n}$ is its adjoint subspace.

Substitution of (12) into (10) and using (11) yields

$$
\mathcal{M} \mathbf{R}_{n} \frac{d \mathbf{a}}{d t}+\mathcal{J} \mathbf{R}_{n} \mathbf{a}+\mathcal{N}\left(\mathbf{R}_{n} \mathbf{a}, \mathbf{R}_{n} \mathbf{a}\right)=0 .
$$

Projection onto the left-hand eigenbasis $\mathbf{L}_{n}$ and the use of the bi-orthogonality relation (14), results in the set of coupled amplitude equations,

$$
\frac{d \mathbf{a}}{d t}-\mathbf{S a}+\mathbf{n}(\mathbf{a}, \mathbf{a})=\mathbf{0} .
$$

The operators in the projected system are defined as

$$
\begin{aligned}
& \mathbf{S}=\mathbf{L}_{n}^{H} \mathcal{J} \mathbf{R}_{n}, \\
& \mathbf{n}(\mathbf{a}, \mathbf{a})=\mathbf{L}_{n}^{H} \mathcal{N}\left(\mathbf{R}_{n} \mathbf{a}, \mathbf{R}_{n} \mathbf{a}\right) .
\end{aligned}
$$

In terms of the individual components, the evolution of amplitude $a_{j}(t)$ is governed by

$$
\frac{d a_{j}}{d t}-\sum_{k=1}^{n} b_{j k} a_{j k}+\sum_{k=1}^{n} \sum_{l=1}^{n} c_{j k l} a_{k} a_{l}=0, \quad j=1, \ldots, n .
$$

The coefficients in the projected system are defined as

$$
\begin{aligned}
b_{j k} & =\mathbf{l}_{j}^{H} \mathcal{J} \mathbf{r}_{k}, \\
c_{j k l} & =\mathbf{l}_{j}^{H} \mathcal{N}\left(\mathbf{r}_{k}, \mathbf{r}_{l}\right) .
\end{aligned}
$$

While this approach is fairly standard, the domain in parameter space where a close correspondence exists between the dynamical behavior contained in (19), for a chosen value of $n$, and the full model (9) is a priori unclear. The selection of the appropriate eigenmodes turns out to be highly problem dependent. For the study of the QG dynamics of the wind-driven circulation, the choice of the basis will be discussed in Sec. IV A.

\section{DYNAMICS OF THE FULL MODEL}

As is usual in studies of dynamical systems with several parameters, a set of standard parameter values is chosen. These values (of the dimensional and nondimensional quantities) are shown in Table I and are representative of a basin
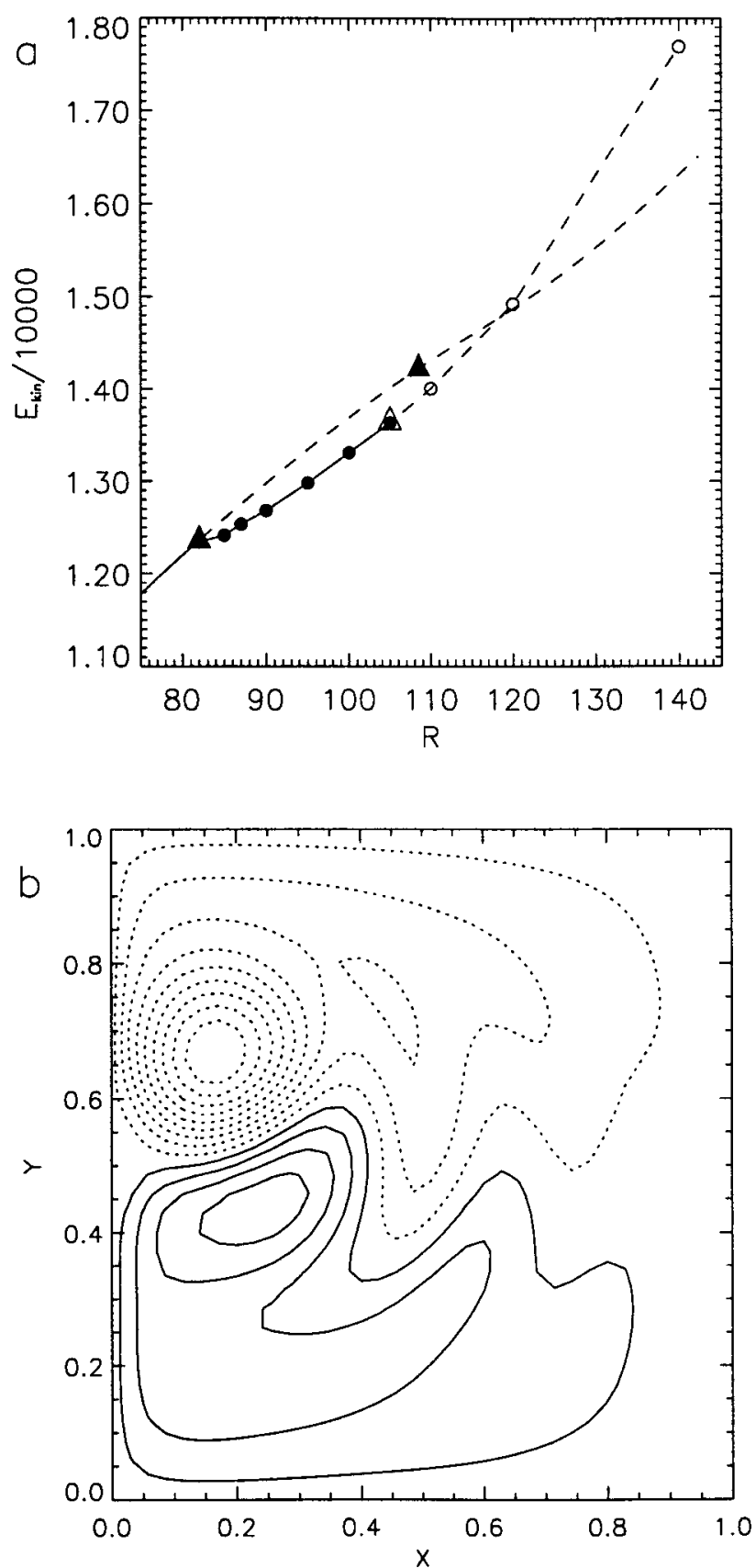

FIG. 1. (a) Part of the bifurcation diagram of the full model. The branch of (un)stable steady state solutions has been indicated by a solid (dashed) line. At the primary Hopf bifurcation, near $R=82$, indicated by a filled triangle, a branch of periodic solutions is spawned from the steady state, with filled (open) circles indicating (in)stability. The Neimark-Sacker bifurcation occurs near $R=105$ (open triangle). (b) Pattern of the steady state streamfunction at $R=82$, close to the first Hopf-bifurcation point (maximum amplitude $=1.783$ ).

of size $1000 \times 1000 \mathrm{~km}$ on a midlatitude $\beta$-plane centered around $45^{\circ} \mathrm{N}$. In this study, only the Reynolds number $R$ is used as control parameter, while the other parameter values will remain fixed at their standard values. The resolution used is $N=36, M=36$, which was shown to be sufficient to capture all bifurcation points accurately. ${ }^{12}$ All results presented below have been obtained by applying an asymmetric windstress forcing with $a=0.2$ in (4).

In Fig. 1(a), part of the bifurcation diagram is shown as 


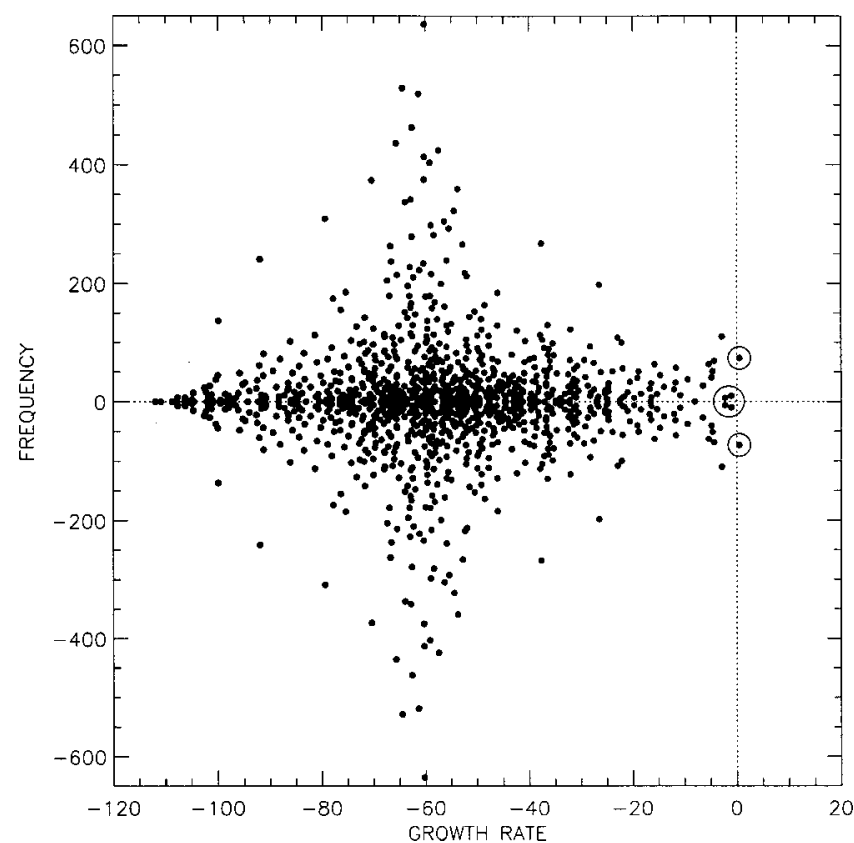

FIG. 2. Spectrum of the linearized operator of the full model at $R=82$, slightly beyond the first Hopf bifurcation. A value of $\sigma_{i}=12.6$ corresponds to 1 year period of the oscillation. The modes of interest have been denoted by circles: the Rossby basin modes $\left(\sigma_{i}= \pm 73\right)$ and the low frequency gyre modes.

a plot of the mean kinetic energy $E_{\text {kin }}$, defined by

$$
E_{\text {kin }}=\frac{1}{2} \int\left(u^{2}+v^{2}\right) d^{2} x
$$

versus $R$. The solid (stable) and dashed (unstable) curves in Fig. 1(a) have been computed with the continuation method and each point represents a steady state. The steady flow becomes unstable through a Hopf bifurcation at $R=81.5$, the location which is marked with a triangle in Fig. 1(a). The steady geostrophic stream function $\psi$ [Fig. 1(b)] at $R=81.5$ shows a typical asymmetric double gyre flow, with a stronger subpolar gyre. The unstable steady state continues to exist over the computational domain [the dashed curve in Fig. 1(a)] and a second Hopf bifurcation occurs at $R=108$ (again labeled with a triangle).

The complete spectrum of the Jacobian $\mathcal{J}$ at $R=82$ (Fig. 2 ) shows that there are high-frequency modes, which have oscillation periods in the order of months ( 2 months corresponds to $\sigma_{i}=75$ ), low frequency modes with typical periods of years ( 1 year corresponds to $\sigma_{i}=13$ ) and stationary modes. The real and imaginary parts of the first three eigensolutions are shown in Fig. 3. The pattern, corresponding to the eigenvalue pair in Fig. 2 which just has crossed the imaginary axis, is a well-known Rossby basin mode [Figs. 3(a) and 3(b)], with an oscillation period of two months. ${ }^{12}$ The second [Figs. 3(c) and 3(d)] and third [Figs. 3(e) and $3(\mathrm{f})]$ pair of modes are so-called gyre modes and characterized by their relatively low frequencies. ${ }^{29}$ In the pattern of the real part of these eigenmodes [Figs. 3(c) and 3(e)], the maximum positive stream function perturbation is located near $(x, y)=(0.2,0.55)$, which is just in the region of maximum velocities of the eastward jet of the basic state [Fig.
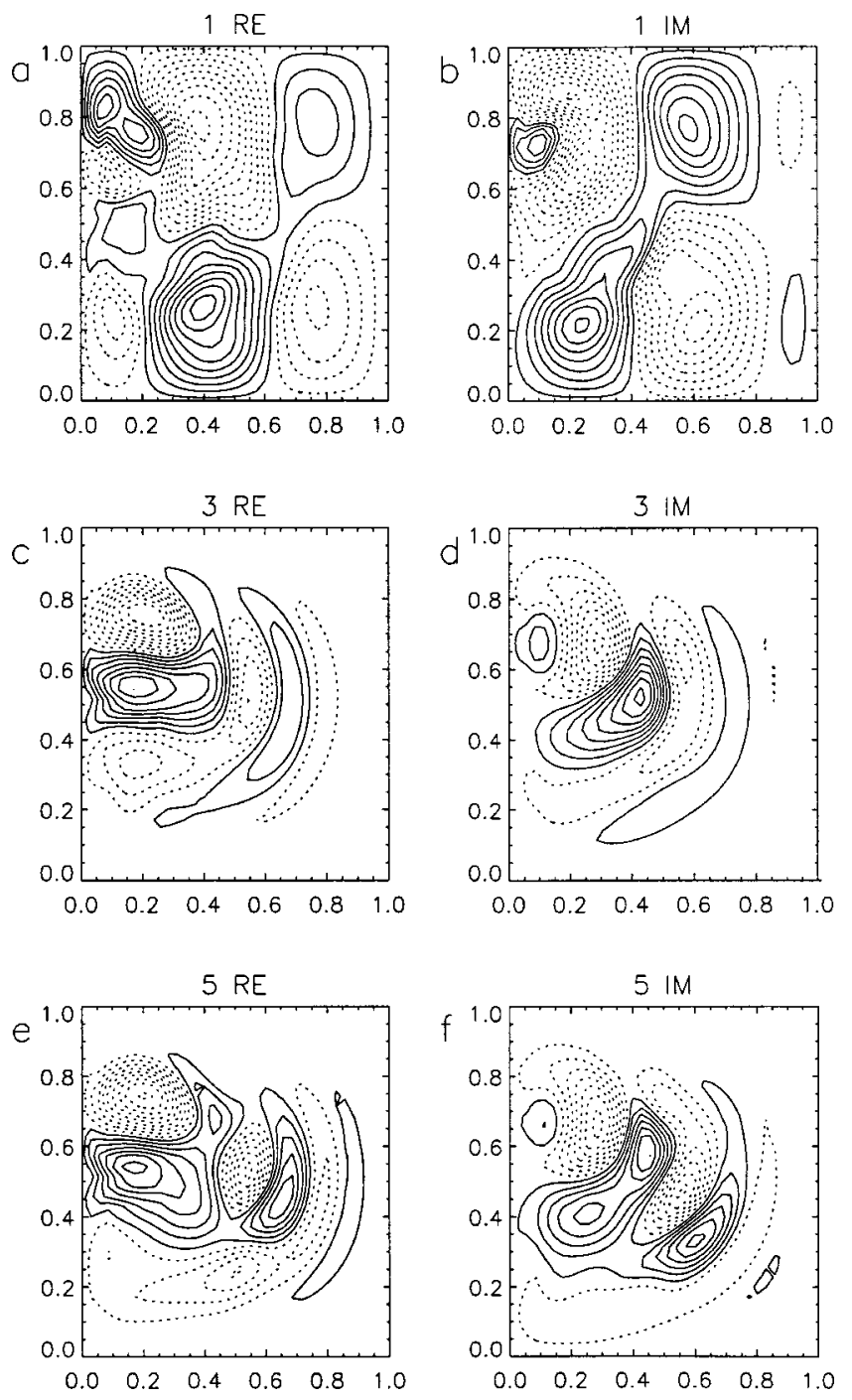

FIG. 3. Stream-function patterns of the leading eigenmodes at the primary Hopf bifurcation. (a) and (b) Real and imaginary part of unstable Rossby basin mode, with frequency $\sigma_{i}=73$ ( $\approx 6$ cycles per year). (c) and (d) Real and imaginary part of the first gyre mode, with frequency $\sigma_{i}=9.8$, corresponding to $\approx 0.8$ cycles per year. (e) and (f) Real and imaginary part of the second gyre mode. Its frequency, $\sigma_{i}=6.2$, corresponds to $\approx 0.5$ cycles per year.

1(b)]. The patterns of both gyre modes hardly differ; in the second gyre mode, there is a secondary positive maximum in the recirculation region of the basic state [near $(x, y)$ $=(0.6,0.4)$ in Fig. 3(e)]. The physics of propagation and growth of both the (destabilized) Rossby basin modes ${ }^{12}$ as well as the gyre modes is now understood. ${ }^{29}$ As its name indicates, the propagation mechanism of the Rossby basin mode is potential vorticity conservation on the $\beta$ plane (midlatitude Rossby-wave dynamics). Here the Rossby basin mode is excited, as it taps into the relative vorticity near the western boundary available through the presence of the confluence of the double gyre flow. However, the propagation mechanism of the gyre modes is independent of $\beta$-plane dynamics and is related to symmetry breaking/restoring tendencies, as will be explained in more detail below. Both modes become more unstable, when the horizontal shear of the background gyre flow is large enough. 

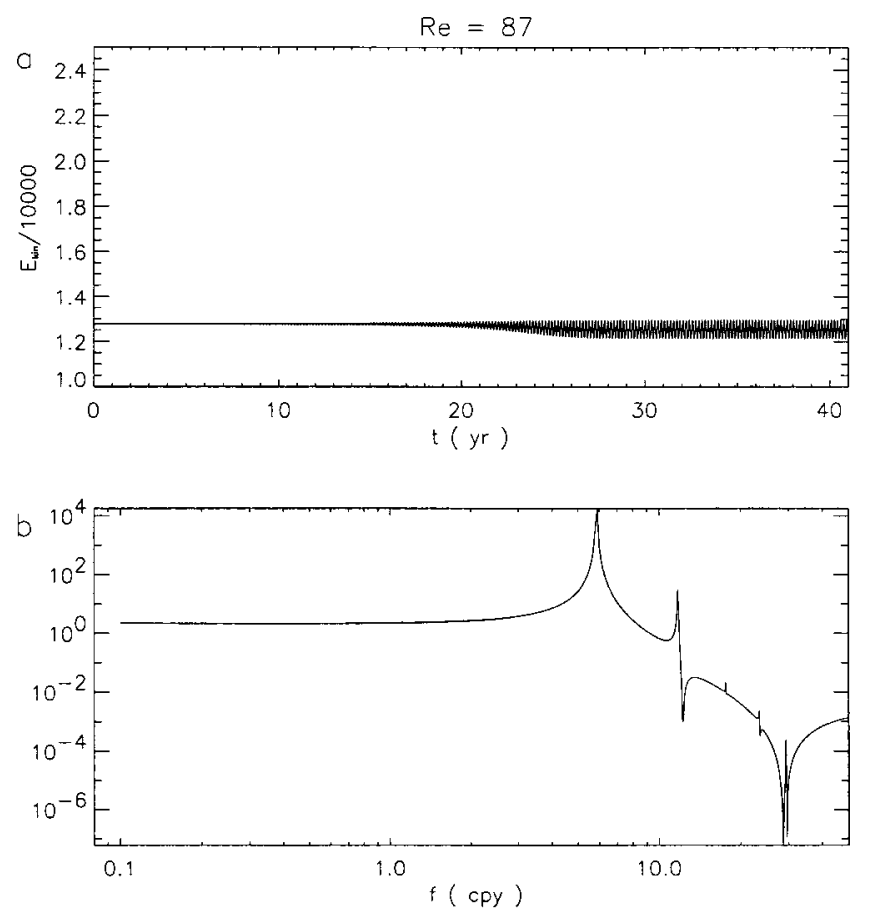

FIG. 4. (a) Time evolution of the basin integrated kinetic energy for $R$ $=87$. (b) Power spectrum of the last 10 years of the time series shown in (a) with the frequency in cycles per year.

The branch of periodic solutions arising from the first Hopf bifurcation at $R=82$ in Fig. 1(a) has been determined from the computation of the time-dependent flows using a Crank-Nicholson time discretization and a timestep of 1 day. The solid curve represents stable periodic solutions and the values of $R$ for which actual flows are computed are labeled by dots. For each solution, the time-averaged value of the kinetic energy is plotted in Fig. 1(a). The time series of the kinetic energy of the stable periodic flow, obtained for $R$ $=87$ is plotted in Fig. 4(a) together with its Fourier spectrum (of the last 10 years of output) in Fig. 4(b). The period of this orbit is about 2 months and the secondary peak in the spectrum is a harmonic of the dominant frequency. The amplitude of this limit cycle increases with increasing $R$ [Fig. 1(a)], but the frequency is nearly independent of $R$ and corresponds closely to that of the Rossby basin mode at the primary Hopf bifurcation in Figs. 3(a) and 3(b).

Three subsequent time series of the flow are shown in Figs. 5 and 6 , at $R=110, R=120$, and $R=140$, respectively, of which the mean kinetic energy is plotted as open circles in Fig. 1(a). The time-dependent behavior is quasiperiodic at $R=110$, as a second, lower frequency appears in the time series [Figs. 5(a) and 5(b)]. This second frequency is about one year and corresponds well with that of the gyre mode in Figs. 3(c) and 3(d). Hence, there is a transition from a stable periodic orbit to quasiperiodic behavior somewhere between $R=105$ and $R=110$. This new frequency is incommensurate with that of the periodic orbit. In other words, it is not a rational multiple of the original (fundamental) frequency. In dynamical systems theory, a transition with these characteristics is usually associated with a secondary Hopf- or Neimark-Sacker bifurcation. ${ }^{23,27}$
From a Fourier transform of the full model streamfunction fields, the spatial pattern associated with this second frequency $\sigma_{i}=13.5$ (about 11 months), is plotted in Figs. $5(\mathrm{c})$ and 5(d). This pattern shows quite a good resemblance with that of the first gyre mode [Figs. 3(c) and 3(d)], which strongly suggests that the gyre mode causes the destabilization of the periodic orbit.

The flow remains quasiperiodic at $R=120$ [Figs. 6(a) and $6(\mathrm{~b})$ ] while the amplitude variation of the kinetic energy becomes larger. A comparison of the spectral characteristics in Fig. 6(b) with those in Fig. 5(b) shows that the harmonics of the gyre mode become more energetic. For $R=140$, the time series of the kinetic energy [Fig. 6(c)] displays irregular variability of large amplitude, with low-frequency oscillations between high- and low-energy states. Correspondingly, the power spectrum is broad banded, although high energy levels can still be recognized at the Rossby basin mode frequency [6 cycles per year (cpy)] and, to a lesser extent, at gyre mode frequencies [ $<1$ cpy, Fig. 6(d)].

From these results of the full model the basic question dealt with in this paper can be specified. Can one show that the high-frequency limit cycle indeed destabilizes through a Neimark-Sacker bifurcation, as suggested from the results above? Once this has been accomplished, can one determine the physical mechanism of destabilization of the limit cycle? To investigate the destabilization of the periodic orbit, one needs to study the linear stability properties of the flow linearized along the proper periodic solution, but this is unfeasible on the original system of partial differential equations. In the following section, the locally reduced model, as derived in Sec. II C, will be used to accomplish this.

\section{ANALYSIS OF THE LOW ORDER MODEL}

To understand the sequence of transitions found in the time-dependent behavior above in more detail, a locally reduced model is constructed. Its capabilities to represent the dynamics of the full model are presented in Sec. IV A. In Sec. IV B, bifurcation analysis is performed on the reduced model.

\section{A. Construction of the low order model}

In terms of representing the dynamics of the full model with a minimal number of degrees of freedom, an orthogonal basis would be preferable, in terms of gaining information with the addition of each new degree of freedom. For autonomous (dynamical) systems, techniques for constructing such a basis based on the statistical properties of the underlying system are available, usually referred to as empirical orthogonal functions (EOF), proper orthogonal decomposition (POD) or Karhúnen Loève decomposition (see Ref. 15 for an overview). However, projection on these types of patterns, for example, EOF's, does not provide information on which modes will become unstable, when varying control parameters. The latter complication may be circumvented by adding noise to the system to detect weakly damped modes, but for the computation of both the attractor and its low frequency variability, exceedingly long time integrations are needed. 

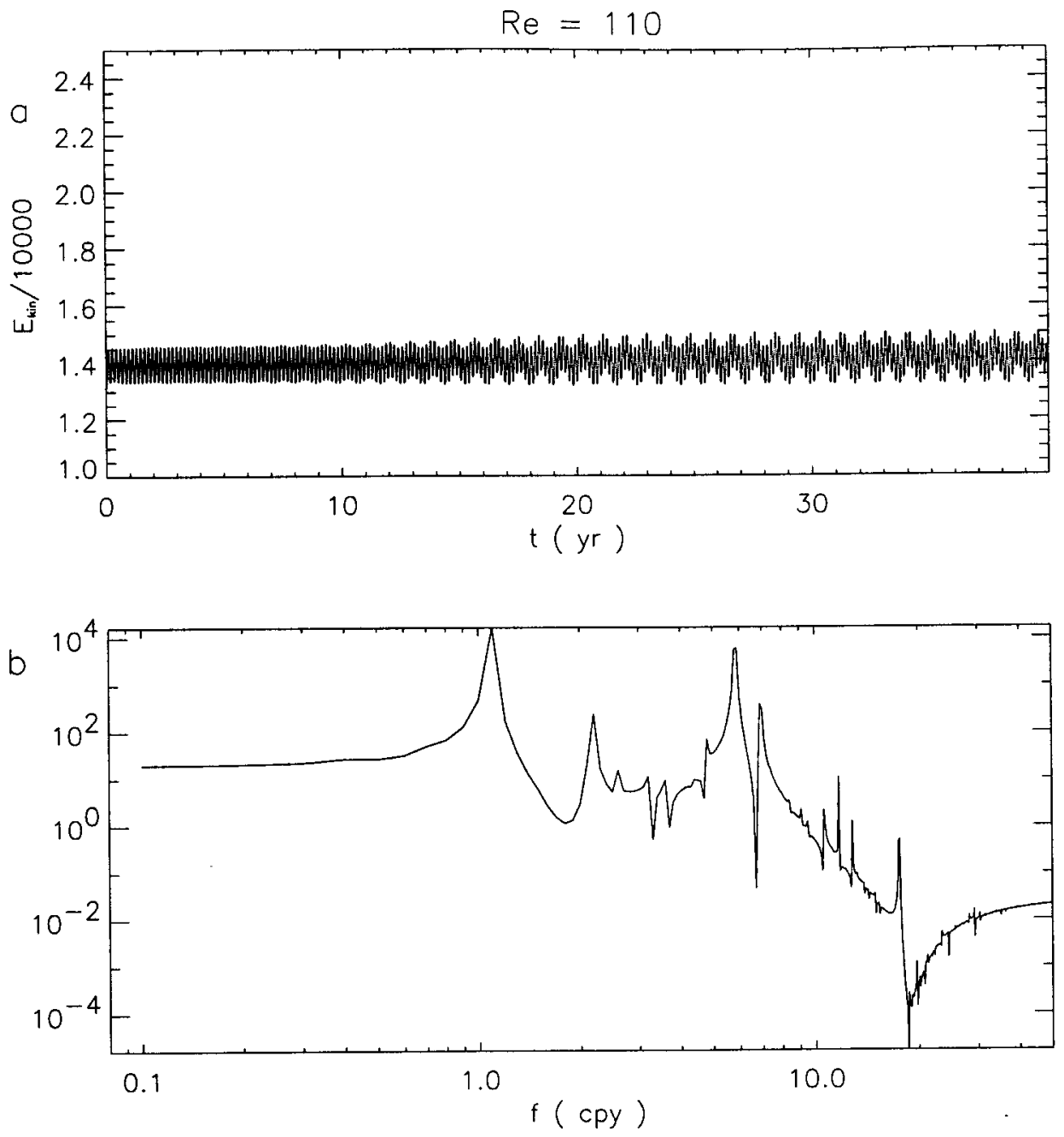

FIG. 5. (a) Time evolution of the basin integrated kinetic energy for $R=110$. (b) Power spectrum of the last 20 years of the time series shown in (a). (c) and (d) Two phases of the unstable mode for $R=110$ at frequency $f$ $=1.05 \mathrm{cpy}$ in (b), obtained from an FFT of the stream-function field of the full model.
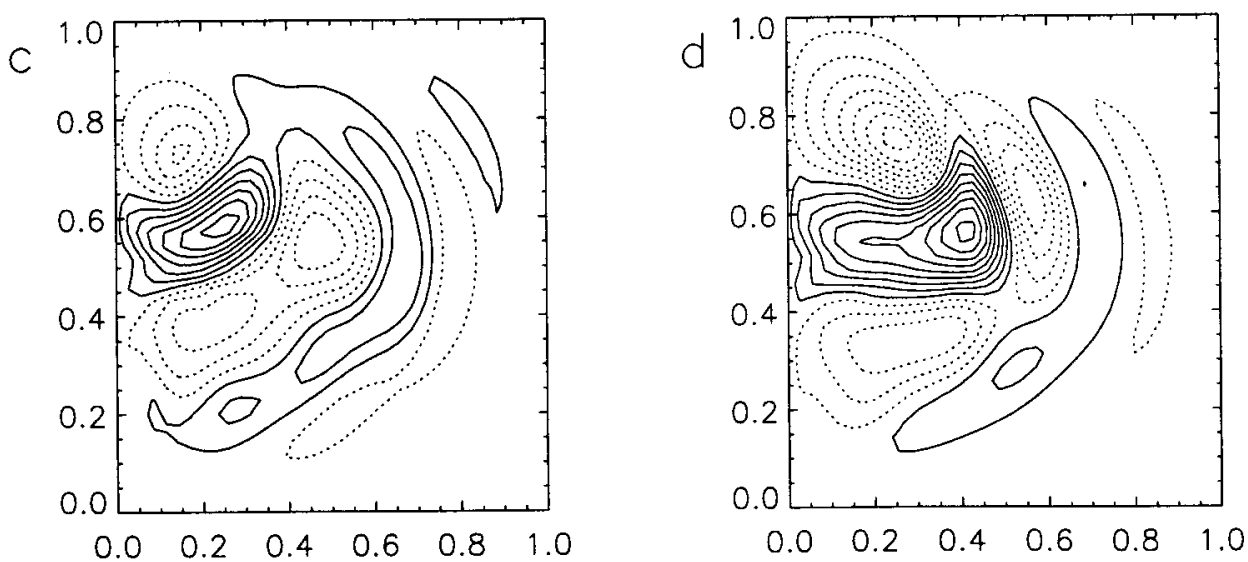

We choose a basis for projection that is spanned by selected modes from the eigenspectrum of the linearized operator near the primary (Hopf) bifurcation, which are explicitly available from the analysis of the full model. This choice is motivated by the appearance of patterns in the flow [Figs. 5(c) and 5(d)] that have a close resemblance to these eigenmodes, even far beyond criticality. As for the use of this eigenbasis, the major drawback is that the eigenmodes are not orthogonal, but only satisfy the bi-orthogonal relation- ship (14). The difficulties that arise from working with a nonorthogonal eigenbasis are addressed in a set of experiments, in which the convergence of a solution of the reduced model is investigated as a function of the number of eigenmodes.

At $R=87$, in a regime where the full model has a stable limit cycle spanned by the Rossby basin mode, the reduced model is constructed (as in Sec. IIC) using the basic steady state (at $R=87$ ) and eigenbasis obtained at $R=82$. Each set 

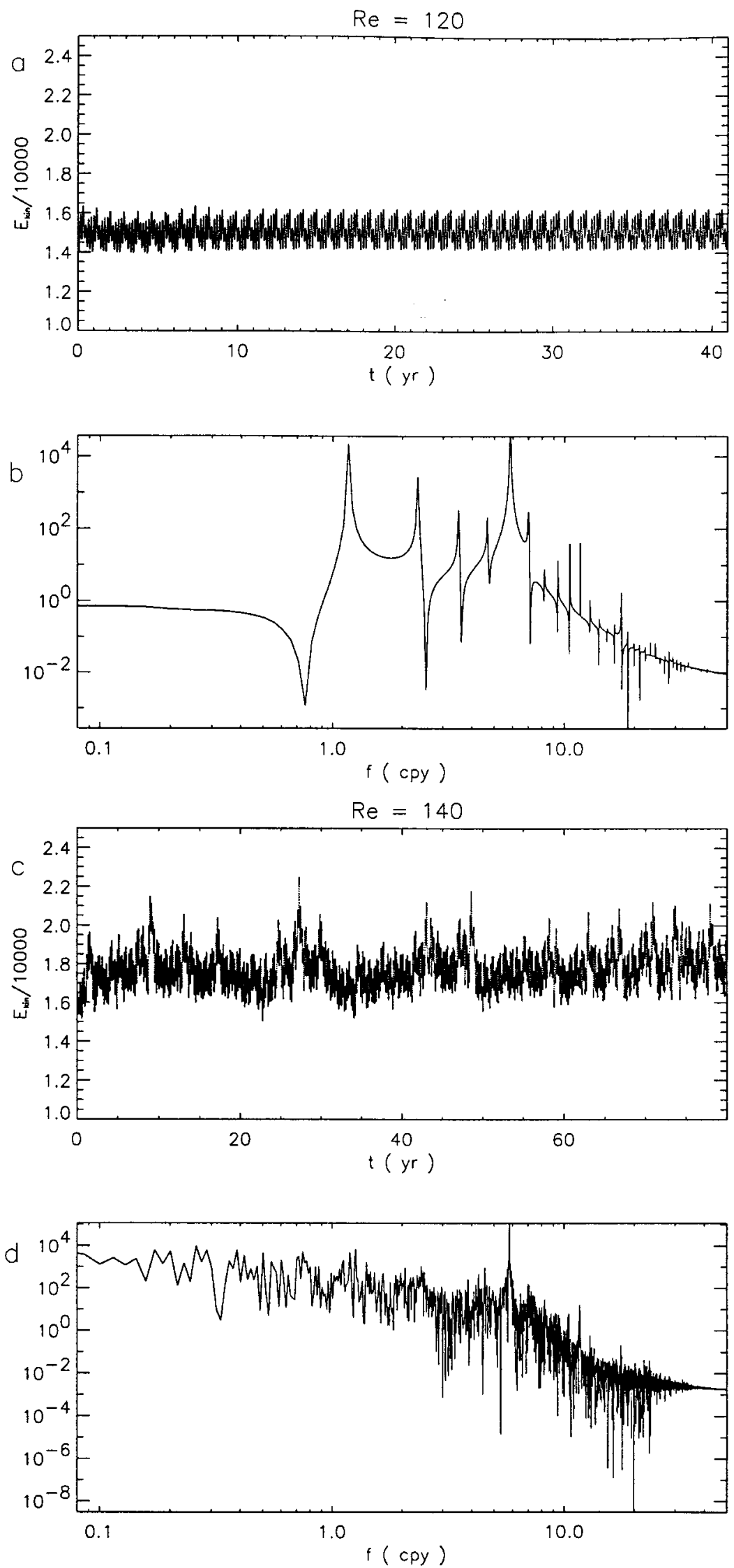

FIG. 6. (a) Time evolution of the basin integrated kinetic energy for $R=120$. (b) Power spectrum of the last 20 years of the time series shown in (a). (c) Time evolution of basin integrated kinetic energy for $R=140$. (d) Power spectrum of the last 70 years of the time series shown in (c). 
TABLE II. Frequency (first row) and amplitudes of selected modes (rows) in three experiments (columns) to test the convergence behavior of the low order model, $R=87$. In all experiments the final state is a limit cycle, as in the full model for this parameter value. $A_{<19}$ represent the remainder of the modes between 6 and 19 .

\begin{tabular}{cccc}
\hline \hline & 1 & 2 & 3 \\
\hline$\omega$ & 73 & 73 & 72 \\
$A_{1}$ & 604.5 & 606.8 & 785.5 \\
$A_{3}$ & 77.7 & 61.1 & \\
$A_{5}$ & 37.7 & 43.7 & \\
$A_{<19}$ & $<40$ & & 292.2 \\
$A_{19}$ & 147.5 & 160.0 & 1271.7 \\
$A_{94}$ & & & \\
\hline \hline
\end{tabular}

of modes includes the unstable eigenmode, whose amplitude is designated by $a_{1}$, and subsequent modes are indicated by $a_{j}, j=2, \ldots, n$. For each truncation $n$, a time integration of the reduced model (19) is performed with initial conditions given by $a_{1}(0)=a_{2}^{*}(0)=100$ and $a_{j}(0)=0, j=3, \ldots n$. For the equilibrium periodic state, a measure for the amplitude of the dynamical modes, i.e.,

$$
A_{j} \equiv \frac{1}{T} \int_{t_{0}}^{t_{0}+T}\left|a_{j}\right| d t
$$

is shown in Table II. In all experiments the attractor is reached in $\left(t_{0}=\right) 50$ years and for $T=20$ years, the $A_{j}$ have converged to their mean values.

When only the unstable Rossby-basin mode and the leading gyre modes (amplitudes $a_{1}, a_{3}$, and $a_{5}$, respectively) as shown in Fig. 3 are included, no finite amplitude solution is found. Such a finite amplitude solution is first found when the stationary mode with amplitude $a_{19}$ is included. The amplitude of all the modes is shown in the first column of Table II, which indicates that the oscillatory modes 1,3 , and 5 plus the stationary mode 19 can give a stable periodic orbit. The remainder of the modes (between 6 and 19) has only relatively small amplitude. When the latter modes are not taken into account, also a periodic orbit is found with slightly changed amplitudes of the modes (second column in Table II). Hence, the smallest reduced model with the same periodic behavior as the full model has 7 degrees of freedom (amplitudes $a_{1}, a_{3}$, and $a_{5}$, and their complex conjugates $a_{2}, a_{4}$, and $a_{6}$, with the stationary mode $\left.a_{19}\right)$.

One can understand why the stationary mode $a_{19}$ is important to be included in the reduced model. It accounts largely for the rectification that arises from the selfinteraction of the unstable Rossby basin mode $\left(\mathbf{r}_{1}\right)$. If we denote this self-interaction by $\mathbf{g}_{0}$, i.e.,

$$
\mathbf{g}_{0}=\mathcal{N}\left(\mathbf{r}_{1}^{*}, \mathbf{r}_{1}\right)+\mathcal{N}\left(\mathbf{r}_{1}, \mathbf{r}_{1}^{*}\right)
$$

then, in the weakly nonlinear regime, the rectification pattern is given by

$$
\phi_{0}=-\mathcal{J}^{-1} \mathbf{g}_{0}=\sum_{n=1}^{r} \frac{\mathbf{l}_{n}^{H} g_{0}}{\sigma_{n}} \mathbf{r}_{n} .
$$
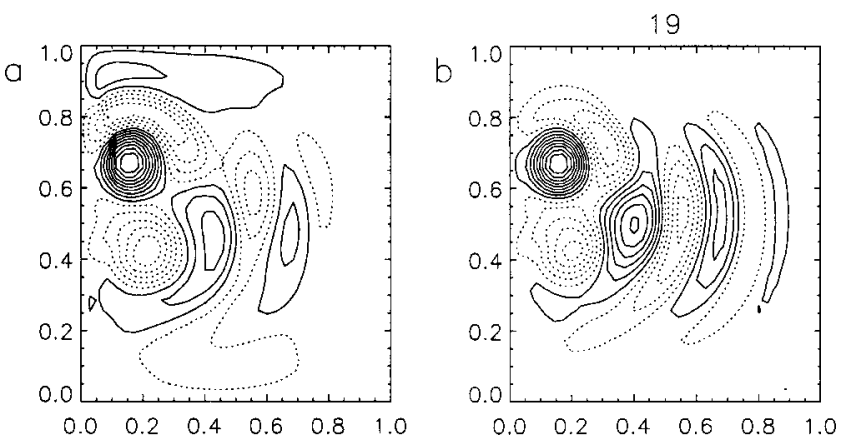

FIG. 7. Comparison of the stream-function patterns of (a) the rectification in the full model (time mean minus basic state) at $R=110$ and (b) the leading stationary eigenmode, residing at position 19 in the spectrum at $R=82$. Both patterns have been scaled by their maximum values.

For the latter expression, the eigenvector representation of the linear operator $\mathcal{J}$ is used, as given by (14). In Fig. 7(a), the stream function of the rectification $\phi_{0}$ is shown, together with the leading stationary mode, residing at position 19 in the eigenspectrum for $R=82\left[\mathbf{r}_{19}\right.$, in Fig. 7(b)]. This mode provides the dominant contribution to the eigenmode expansion in (23).

We want to emphasize here that it is not our aim to derive a low-order model which is able to capture the complete dynamical behavior of the full model. Instead, we only want a local low-order model, which is able to capture the flow transition from periodic to quasiperiodic near $R=105$. Convergence within a reduced model, based on nonorthogonal eigenmodes is unlikely to be accomplished. Expanding the system with additional stationary modes, for instance mode $\mathbf{r}_{94}$, gives worse results in terms of convergence of the projection amplitudes (third column of Table II). This mode provides an additional contribution to the rectification pattern, but it also projects into directions that are dynamically not important. These must in turn be compensated by other nonorthogonal modes, residing deeper in the spectrum.

\section{B. Bifurcation analysis of the reduced model}

The reduced seven-mode model as constructed above is subjected to a bifurcation analysis by varying the control parameter $R$, similar to the full model. Periodic solutions to the set of autonomous equations (18a), with unknown period $T$, are computed by continuation. In (18a), the period $T$ introduces an additional degree of freedom ${ }^{30}$ to the system associated with the freedom of phase [if $\mathbf{a}(t)$ is a solution, then for arbitrary $t_{0}, \mathbf{a}\left(t+t_{0}\right)$ is also a solution]. To remove this degree of freedom, we have chosen to constrain the real part of one of the systems components $\left(a_{q}+a_{q}^{*}\right)$ at time $t_{0}$ to a fixed value $Q$. It has been verified that solutions do not depend on the choice of both $t_{0}$ and $Q$. Periodic boundary conditions in time are applied and the resulting system of ordinary differential equations becomes

$$
\begin{aligned}
& \frac{d \mathbf{a}}{d t}-\mathbf{S a}+\mathbf{n}(\mathbf{a}, \mathbf{a})=\mathbf{0}, \\
& \mathbf{a}(0)=\mathbf{a}(T),
\end{aligned}
$$




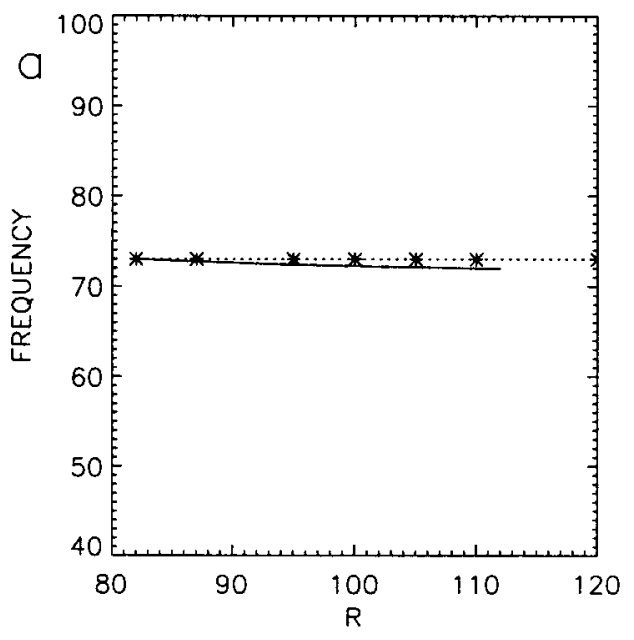

$a_{q}\left(t_{0}\right)+a_{q}^{*}\left(t_{0}\right)=Q$.

Using a Crank-Nicholson scheme, (24) is discretized in time and a Newton-Raphson method is applied to solve the resulting set of nonlinear algebraic equations. For the actual numerical computation, it is convenient to rescale time with $T$, resulting in (24) being defined on the $[0,1]$ interval for all values of $T$. The Newton iteration is initiated from a weakly nonlinear approximation to the solution or from a solution obtained by time integration of the reduced model. Once a periodic solution is found, it can be followed as a function of the system parameters through continuation. ${ }^{28}$ The use of 40 grid points in the time domain suffices, since doubling the temporal resolution to 80 grid points resulted in changes of amplitudes, and the frequency of the limit cycle of less than $1 \%$.

The frequency of the limit cycle found within the reduced model versus $R$ is plotted in Fig. 8(a), together with the amplitude of the modes in Fig. 8(b). In Fig. 8(a), also the dominant frequencies determined from the time integrations of the full model are marked, demonstrating the capabilities of the reduced model to describe the periodic orbit. With increasing $R$, the amplitudes of all modes increase. The mode with amplitude $A_{19}$ is quite large over the whole parameter range, indicating that rectification process generated by selfinteraction of the Rossby basin mode are fairly strong.

To compute the stability of the limit cycle, the reduced model equations are linearized along the periodic orbit $\mathbf{A}(t)$, obtained from solving (24), i.e.,

$$
\hat{\mathbf{a}}_{t}-(\mathbf{S}+\mathbf{n}(\mathbf{A}, .)+\mathbf{n}(., \mathbf{A})) \hat{\mathbf{a}}=\mathbf{0} .
$$

Information about the linear stability of the periodic orbit is contained in the eigenvalues of the monodromy matrix, ${ }^{30}$ the so-called Floquet multipliers, $\rho_{m}$. This monodromy matrix is defined as the fundamental solution to (25) evaluated at $t$ $=T$ and is computed by integrating all possible initial conditions, conveniently represented by the identity matrix, over one cycle of oscillation of the periodic orbit. If one or more Floquet multipliers lie outside the unit circle in the complex plane, the periodic solution is an unstable limit cycle. If, on the other hand, all multipliers lie inside the unit circle, the limit cycle is stable. For autonomous systems like (24), one
Floquet multiplier associated with the periodic solution $\mathbf{A}(t)$ always equals unity. This implies that perturbations along a direction tangent to the periodic orbit neither grow nor decay. This property also provides an important and necessary check on the numerical computation of the stability of the periodic orbit.

The full temporal and spatial structure of the Floquet modes is given by $\mathbf{V}_{m}(\mathbf{x}, t) e^{\gamma_{m} t}$, where $\mathbf{V}_{m}(\mathbf{x}, t)$ is the $m$ th Floquet eigenvector and $\gamma_{m}=\log \left(\rho_{m}\right) / T$ is the associated Floquet exponent. The temporal structure of eigenmode $\mathbf{V}_{m}$ is obtained by integrating (25) over one cycle, starting from the eigenvector of the monodromy matrix associated with the Floquet multiplier $\rho_{m}$. There are three distinct scenarios associated with the loss of stability of the periodic orbit as a control parameter is varied. A Floquet multiplier leaving the unit circle at +1 , results in either a transcritical, a symmetrybreaking or a cyclic fold bifurcation. This is analogous to the bifurcations of time-independent flows (see, for instance, Ref. 27). When a multiplier leaves through -1 , a period doubling or flip bifurcation takes place. Finally, when two complex conjugate eigenvalues exit the unit circle away from the real axis, a Neimark-Sacker bifurcation occurs. This introduces a new, possibly incommensurate, frequency into the system, similar to the primary Hopf bifurcation.

The Floquet multipliers and exponents are plotted in Figs. 9(a) and 9(b) and near 0.7 $\pm 0.71 i$ [in Fig. 9(a)] a pair of Floquet multipliers leaves the unit circle. This indicates that a Neimark-Sacker bifurcation occurs at $R=110.1$. This value is slightly above that guessed from the full model. The limit cycle is shown (anomalies with respect to the unstable steady state at $R=110.1$ ) for two phases in Figs. 9(c) and 9(d) and again the Rossby basin mode character of the solution is apparent. The dimensionless frequency, associated with the unstable Floquet vector, is 9.01 (corresponding to about 16 months), to be compared with 13.8 (11 months) in the full model. The Floquet vector is shown in Figs. 9(e) and 9(f) and its spatial structure and frequency is strongly related to the leading gyre modes in Figs. 3(c)-3(f).

In Figs. 9(a) and 9(b) the gyre modes (indicated by GM1 and GM2, respectively) nearly merge for increasing values of the control parameter $R$, as can be witnessed from the fact 

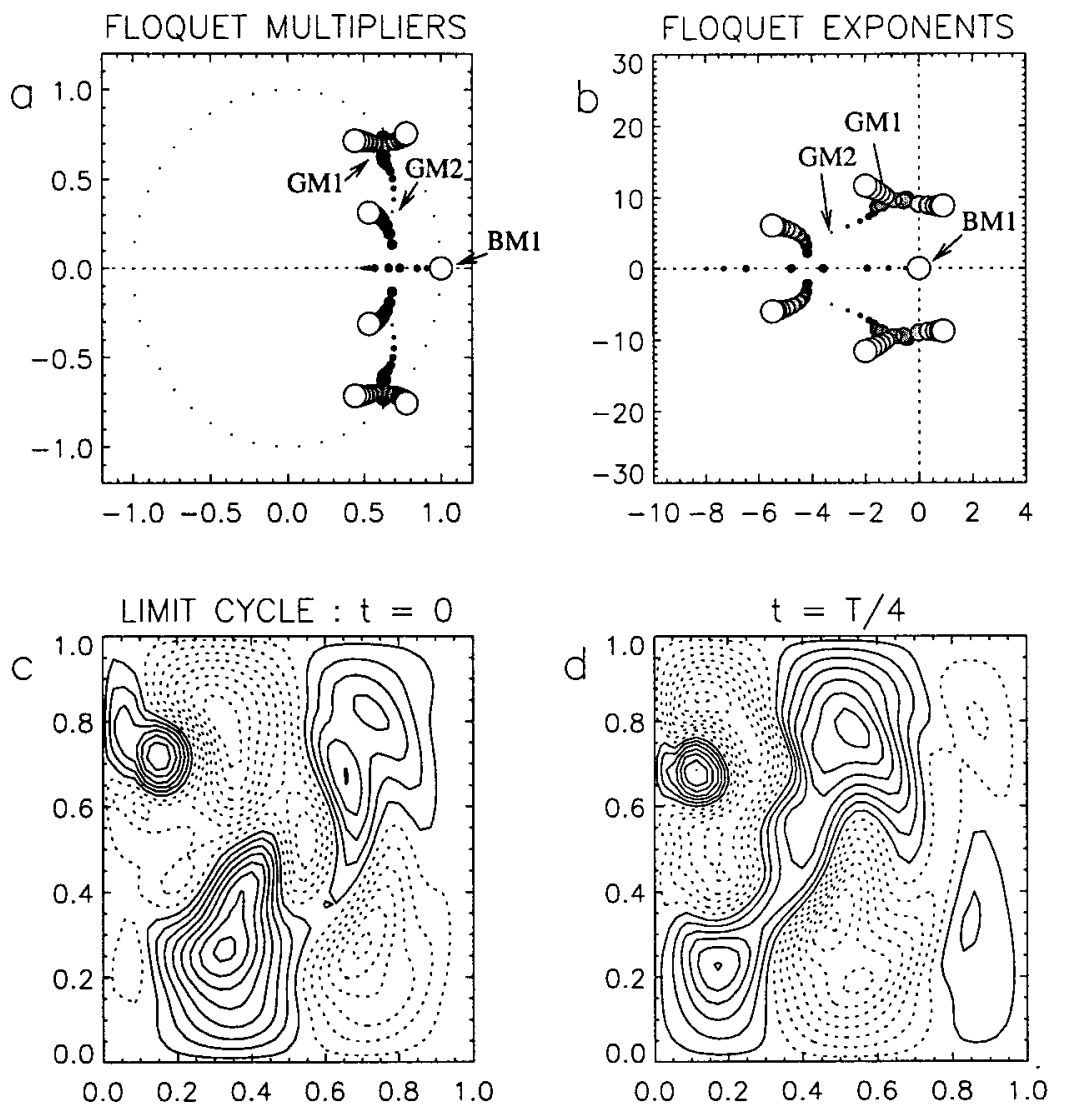

FIG. 9. Linear stability of the limit cycle in the low order model. (a) Floquet multipliers, $\rho_{m}$, as a function of $R$. The unit circle in the complex plane is indicated by the dotted line and separates a stable $\left(\left|\rho_{m}\right|<1\right)$ from an unstable $\left(\left|\rho_{m}\right|>1\right)$ limit cycles. (b) Floquet exponents, $\gamma_{m}=\log \left(\rho_{m}\right) / T$, as a function of $R$. Floquet exponents in the left (right) half-plane indicate (un)stable eigenvectors. Small dark (large light) dots correspond to low (high) Reynolds numbers $[R \in(82.0,120.0)]$. GM1 denotes the position of the first gyre mode, GM2 of the second gyre mode and BM1 that of the leading Rossby basin mode. (c) and (d) Spatial pattern of the limit cycle at $R=110.1$, with respect to the steady state at $R=110.1$ at $t=0$ and $t=T / 4$. (e) and (f) Spatial pattern of two phases of the leading Floquet vector.
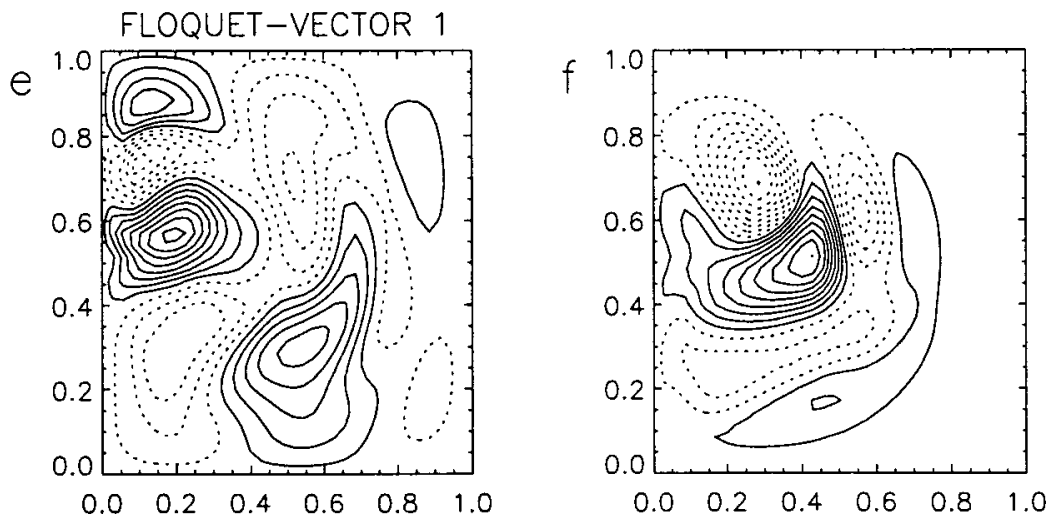

that their eigenvalues become almost identical. This suggests that both gyre modes are indispensable in setting up the spatial pattern of the unstable mode at the Neimark-Sacker bifurcation. This is confirmed by experiments where either one of the gyre modes has been omitted from the basis of the reduced model. In both cases no bifurcations occur before $R=160$ and no gyre mode becomes unstable.

Although the correspondence of the reduced and full model up to the Neimark-Sacker bifurcation is good, the reduced model turns out to be capable of representing flows at higher values of $R$. For $R=120$, the projection coefficients of the trajectory of the full model onto the modes of the reduced model are shown in Fig. 10. This indicates that the limit cycle is represented by $\mathbf{r}_{1}$, its rectification by $\mathbf{r}_{19}$ and that the low-frequency signal is represented both by $\mathbf{r}_{3}$ and $\mathbf{r}_{5}$. Having established the correspondence of the dynamics of the reduced and the full model, we will use both the pe- riodic state and the Floquet vectors of the reduced model for the description of the physical mechanism associated with the Neimark-Sacker bifurcation in the next section.

\section{PHYSICAL MECHANISM OF THE SECONDARY INSTABILITY}

To describe the physical mechanism of the destabilization of the (Rossby basin mode) limit cycle by the (gyre) Floquet mode, it is necessary to recapitulate the essential physics of the destabilization of the gyre mode on the steady state flow.

The oscillatory behavior of the gyre mode is characterized by an almost standing wave pattern which propagates perpendicularly to the direction of the eastward jet [Figs. 3(c)-3(f)]. In the barotropic quasigeostrophic model of the double gyre flow, with an antisymmetric wind-stress pattern 


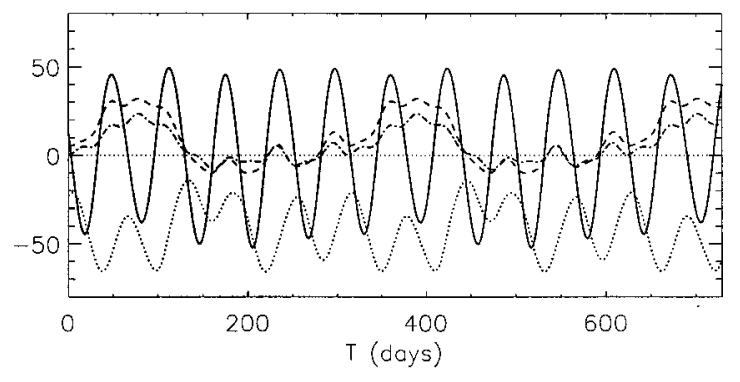

FIG. 10. Evolution of the projection coefficients of the eigenmodes in the full model at $R=120$ : Rossby basin mode ( $\mathbf{r}_{1}$, solid line), leading gyre modes $\left(\mathbf{r}_{3}\right.$, dashed, and $\mathbf{r}_{5}$, dashed-dotted line) and the first real mode $\left(\mathbf{r}_{19}\right.$, dotted line).

$[a=0$ in (4)], this mode arises through merging of two nonoscillatory modes. ${ }^{29}$ The first one, called a $P$ mode, consists of a tripole which is symmetric with respect to the midaxis of the basin; its effect is to increase the shear of the eastward jet. In the case $a=0$, this mode was shown to be related to the appearance of multiple steady states through a symmetry-breaking pitchfork bifurcation. The spatial pattern of the second stationary mode, called $L$-mode and related to the occurrence of limit points, is antisymmetric and dipolar and its effect is to strengthen or weaken both gyres. These two real modes tend to deform on the asymmetric branches of solutions in such a way that they merge to become oscillatory: a gyre mode results from this marriage. The growth and decay of the energy of the gyre mode in the different stages of the oscillation is determined by a pure shear mechanism. The combined effect of this shear, the asymmetry of the steady flow and the symmetry-breaking mechanism, which induces temporal constraints between the symmetric and antisymmetric components of the perturbations, cause the low-frequency oscillation. Once the steady state becomes more asymmetric, the gyre mode becomes more unstable because it can feed on the increased horizontal shear.

The physical mechanism of the destabilization of the limit cycle is analyzed within the reduced model, since Floquet vectors and the limit cycle are available near criticality. As the asymmetry in the strength of both the gyres is expected to play a major role in the destabilization of the limit cycle, a measure of this asymmetry $\mathcal{S}_{a}$ was chosen as the difference between the maximum stream function values of the subtropical $\left(\psi_{\mathrm{st}}\right)$ and subpolar gyre $\left(\psi_{\mathrm{sp}}\right)$, i.e., $\mathcal{S}_{a}=\psi_{\mathrm{st}}$ $-\psi_{\text {sp }}$. To monitor the interaction between the perturbation (i.e., the Floquet vector) and the limit cycle, changes in the perturbation kinetic energy were calculated. The energy balance of the perturbation can be written as

$$
\frac{d e_{\mathrm{kin}}}{d t}=\widetilde{\mathcal{I}}-R^{-1} \widetilde{\mathcal{D}}
$$

where $\widetilde{\mathcal{I}}$ is the Reynold's stress energy production and $\widetilde{\mathcal{D}}$ is the dissipation. The latter depends only on the perturbation structure, whereas the energy production both depends on the periodic background state as well as the perturbations.

For three cases near critical conditions, $R=102.8, R$ $=110.1$, and $R=117.2$, the total kinetic energy of the limit cycle is plotted versus $\mathcal{S}_{a}$ in Figs. 11(a)-11(c), respectively.
The labels $A-G$ indicate specific stages along the limit cycle and simultaneously indicate the path taken through this phase-plane picture. For each case, the asymmetry in the limit cycle increases from $H \rightarrow C$ and decreases from $C$ $\rightarrow H$. While the range of asymmetries do not change with increasing $R$, the overall kinetic energy increases. The main change with increasing $R$ is that the phase in which the asymmetry decreases is associated with higher kinetic energy of the total flow.

The frequency of the Floquet mode is lower than that of the periodic orbit. To monitor the growth of this mode, we plot the perturbation kinetic energy $\left(e_{\text {kin }}\right)$ versus its tendency $\left(d e_{\text {kin }} / d t\right)$ in Figs. 11(d)-11(f) again for $R=102.8, R$ $=110.1$, and $R=117.2$. Only the evolution of these quantities over one period of oscillation of the limit cycle is shown. During the phase of high $(B \rightarrow D)$ asymmetry of the limit cycle, the Floquet mode grows. Also in the phase of low asymmetry $(F \rightarrow H)$, there is growth of the Floquet mode, although substantially weaker than during the high asymmetry phase. As can be seen, the total growth increases with $R$, mainly due to an increase in growth rates in the highasymmetry phase $(B \rightarrow D)$. The growth of the Floquet mode appears closely associated with the destabilization mechanisms of the gyre mode in the steady state. Hence, this is a motivation to obtain $P$ - and $L$-mode signatures in the Floquet vector.

The $P$-mode signature of the Floquet vector is constructed at criticality $(R=110.2)$ by looking at the time series of this mode at the point [marked by an asterisk in Fig. 12(a)] where the background gyre flow (at the NeimarkSacker bifurcation) has its maximum. This time series is correlated with time series at all other points and the correlation coefficients are plotted in Fig. 12(a). In the same way, the $L$-mode part of the Floquet vector is determined by looking at the time series of the difference between the maxima of both gyres [marked by the two asterisks in Fig. 12(b)] and correlating this time series with those over the rest of the domain. The correlation coefficients give the pattern in Fig. 12(b). The effect of the $P$ mode on the mean flow is to increase/decrease the shear between the gyres, whereas the $L$ mode tends to strengthen/weaken the gyres, just as in the more idealized $(a=0)$ double gyre problem. ${ }^{29}$ The $P$-mode and $L$-mode amplitudes are plotted versus time in Fig. 12(c). This shows that the $P$ and $L$ mode are nearly in quadrature and they clearly control the low frequency behavior of the flow. Their interaction with the background limit cycle causes growth, with the larger (smaller) peak corresponding to $B \rightarrow D(F \rightarrow H)$ in Fig. 11(e). However, it is difficult to establish a mechanistic picture of the way this growth is coupled to the amplitudes of the $P$ and $L$ mode individually.

When the stability of the time-mean state of the limit cycle is computed, a Hopf bifurcation associated with the destabilization of the first gyre mode is found at $R=142.2$, significantly beyond the value found in both the full model and in the Floquet analysis of the low-order model. This shows that the rectification associated with the Rossby basin mode prevents the destabilization of the gyre mode, as it 

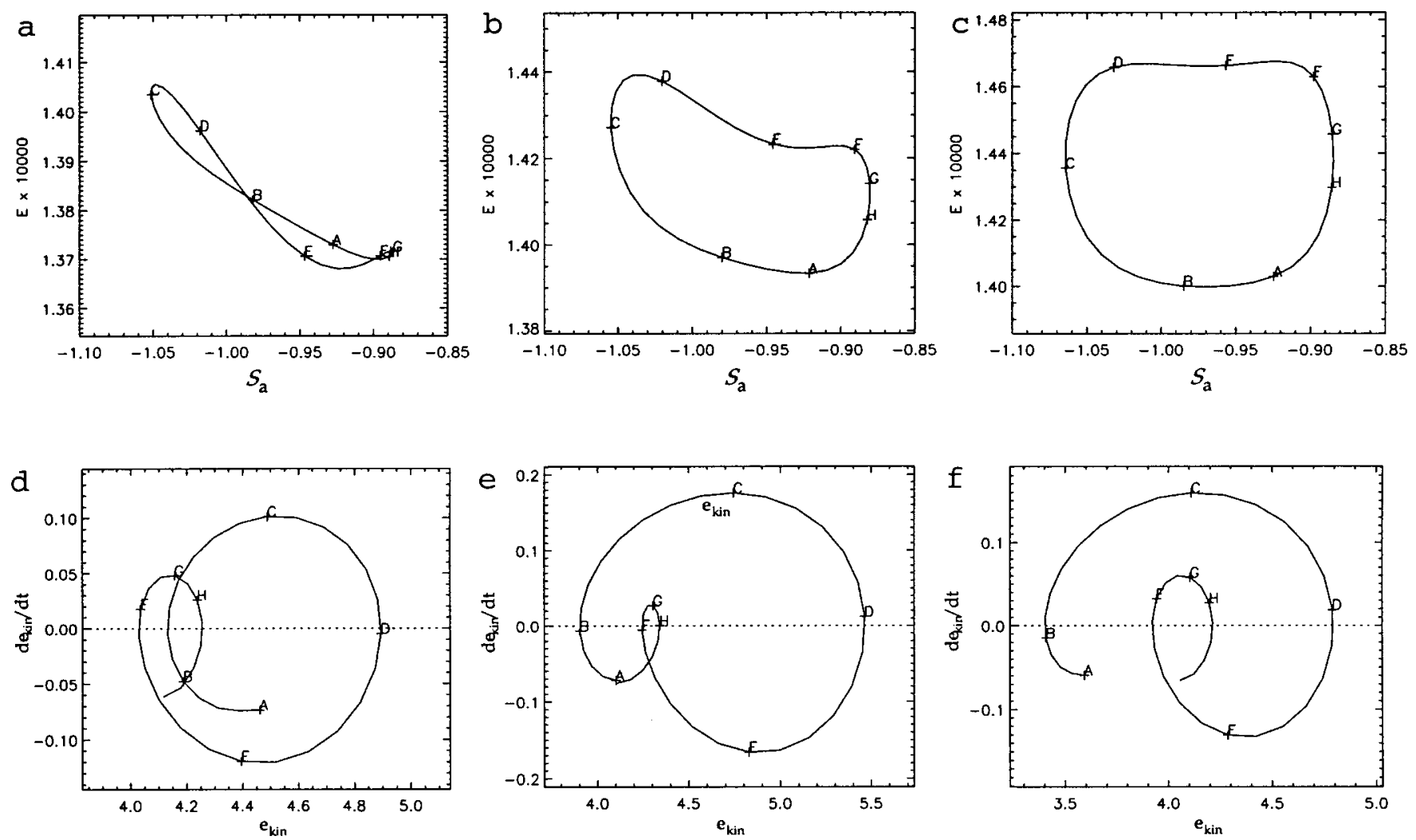

FIG. 11. Phase space diagram of the limit cycle for (a) subcritical conditions $(R=102.8)$, (b) critical conditions $(R=110.1)$, and (c) supercritical conditions at $R=117.2$. The abscissa shows a measure for the asymmetry of the flow, $\mathcal{S}_{a}$, versus the integrated kinetic energy $E_{\text {kin }}$. Phase space views on the evolution of the leading Floquet-vector over one oscillation cycle of the periodic orbit, (d) subcritical conditions, (e) critical conditions, and (f) supercritical conditions.

tends to reduce the asymmetry between the subpolar and the subtropical gyre [Fig. 5(c)]. The temporal changes in the limit cycle are important for the destabilization process. In the absence of gyre modes, the rectification due to the self interaction of the Rossby basin modes stabilizes the mean flow. On the other hand, it is the time-dependent character of the Rossby basin mode which enhances the contrast (and thus the shear) between the gyres with each passing, that counteracts the stabilizing effect due to rectification and renders the gyre mode(s) unstable.

\section{SUMMARY AND DISCUSSION}

In the analysis of the transition to complex timedependent wind-driven ocean flows, one additional step has been taken by determining the physical mechanism of the transition of a high-frequency oscillating flow to a quasiperiodic flow, having both a high- and low-frequency component. The steady wind-driven double-gyre flow becomes unstable through a Hopf bifurcation at relatively low values of the Reynolds number $R$. The physical mechanism of propagation of the critical mode is the same as that of a barotropic Rossby basin mode and its growth is related to the horizontal shear of the background flow field. ${ }^{12}$ It is this barotropic instability, which gives a time-dependent periodic gyre flow which a dominant frequency in the order of months. With increasing values of $R$, this periodic orbit destabilizes through a Neimark-Sacker bifurcation. While this destabilization could be anticipated from the time-dependent flows computed with the full model, the exact details of the destabilization process were captured using a (numerically constructed) low-order model. It is now possible to exploit the versatility of the low-order model and to pinpoint the essential physical modes that govern the variability, as observed in the full model.

The application of the locally reduced model appears to have a large application potential, although one has to realize that its results have to be verified with those of a full model. Ideally one would like to work with a locally reduced model that shows convergence to the full model dynamics as the number of modes is increased. The results summarized in Table II indicate that this property is difficult to satisfy when (nonorthogonal) eigenmodes are used. However, the eigenmodes have the attractive property that they have a direct physical interpretation.

With the current state of continuation methods, already large dimensional dynamical systems can be analyzed (up to $10^{6}$ degrees of freedom, see Ref. 31). There are specific eigenvalue solvers which can locate a specific number of right and left eigenvectors, making a numerical construction of such locally reduced models possible for a large class of applications. ${ }^{32}$ With such models, realizing their limitations, one can analyze one step further into the complexity of flows, by allowing the determination of local periodic orbits and their linear stability. In addition, questions related to the effects of noise on the dynamics can be tackled. While it is very difficult to determine probability density functions for 

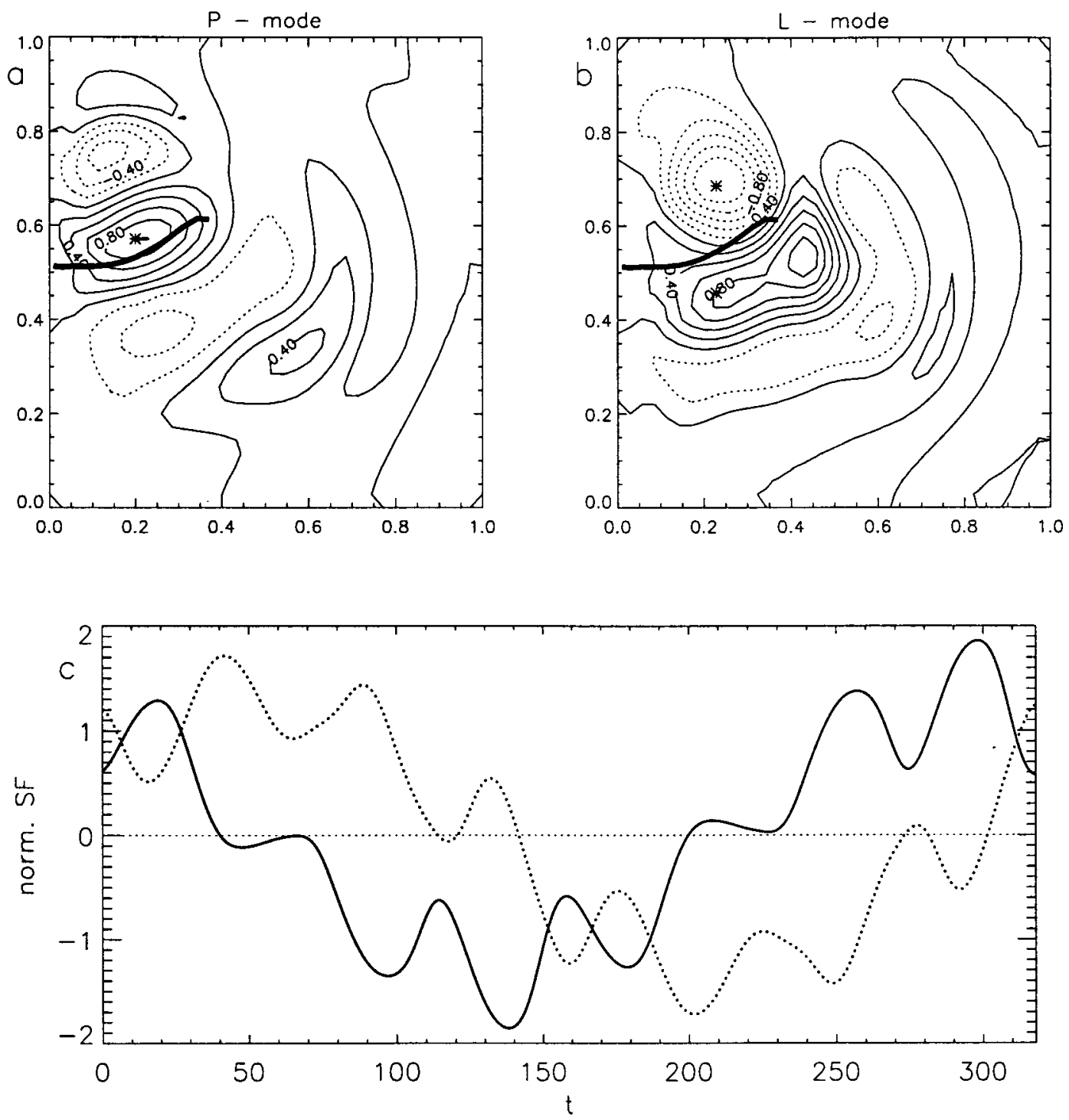

FIG. 12. Regression maps of the (a) $L$ mode and (b) $P$ mode, obtained from the leading Floquet vector at $R=110$, CI is 0.2 . The solid line marks the position of the basic state jet. (c) Time series of the indices of the $P$ mode [dashed line, taken at jet axis at the position marked by $*$ in (a)] and the $L$ mode [solid line, constructed from the difference of the stream-function values of the Floquet mode at the positions marked by $*$ in $(b)]$.

systems of partial differential equations, this can be done for small dimensional systems.

From the reduced model developed here it is shown that the (gyre) Floquet mode can extract energy from the Rossby mode periodic orbit through the changes in the horizontal shear of the background state. The propagation mechanism of the Floquet mode is shown to be the same as that of the gyre mode. ${ }^{29}$ The change in amplitude of a $P$-mode and $L$-mode pattern controls the low-frequency signal of the Floquet mode. With each passing of the Rossby-basin mode the asymmetry of the flow is enhanced and induces a coupling with the amplitudes of the $P$ and $L$ modes which control the growth of the perturbation associated with the Floquet mode (Fig. 12). While this physical mechanism may appear as a detail within a model of very limited applicability, its description is of utmost importance. Work within a hiearchy of models of the ocean circulation has indicated that the gyre mode is a very robust feature which is not limited to pure barotropic quasigeostrophic models in square basins. ${ }^{31}$ Since its dynamics are related purely to the shear of the background western boundary current, and is not related to Rossby wave propagation, its time scale inherently has low frequency. Hence, the results here contribute to the physics of the low-frequency variability of more general ocean flows and eventually to that of the climate system.

\section{ACKNOWLEDGMENTS}

This work was supported by the Netherlands Organization for Scientific Research (NWO) under a PIONIER grant to H.D. All computations were performed on the CRAY C90 at the Academic Computing Center (SARA), Amsterdam, the Netherlands within the project SC498. Use of these computing facilities was sponsored by the National Computing $\mathrm{Fa}-$ cilities Foundation with financial support from NWO. 
${ }^{1}$ H. Stommel, "The westward intensification of wind-driven ocean currents," Trans., Am. Geophys. Union 29, 202 (1948).

${ }^{2}$ W. Munk, "On the wind-driven ocean circulation," J. Meteorol. 7, 79 (1950).

${ }^{3}$ S. Jiang, F.-F. Jin, and M. Ghil, "Multiple equilibria and aperiodic solutions in a wind-driven double gyre, shallow water model," J. Phys. Oceanogr. 25, 764 (1995).

${ }^{4}$ J. C. McWilliams, "Modeling the ocean general circulation," Annu. Rev. Fluid Mech. 28, 215 (1996).

${ }^{5}$ J. D. McCalpin, "The statistics and sensitivity of a double-gyre model: the reduced gravity, quasigeostrophic case," J. Phys. Oceanogr. 25, 806 (1995).

${ }^{6}$ P. S. Berloff and J. C. McWilliams, "Large-scale, low-frequency variability in wind-driven ocean gyres," J. Phys. Oceanogr. 29, 1925 (1999).

${ }^{7}$ S. P. Meacham, "Low frequency variability of the wind-driven circulation," J. Phys. Oceanogr. 30, 269 (2000).

${ }^{8}$ K.-I. Chang, M. Ghil, K. Ide, and C.-C. A. Lai, "Transition to aperiodic variability in a wind-driven double-gyre circulation model," J. Phys. Oceanogr. 31, 1260 (2001).

${ }^{9}$ J. Nauw and H. A. Dijkstra, "On the origin of low frequency modes of the double gyre wind-driven ocean circulation,” J. Mar. Res. 59, 567 (2001).

${ }^{10} \mathrm{P}$. Cessi and G. R. Ierley, "Symmetry-breaking multiple equilibria in quasi-geostrophic, wind-driven flows," J. Phys. Oceanogr. 25, 1196 (1995).

${ }^{11}$ S. Speich, H. A. Dijkstra, and M. Ghil, "Successive bifurcations of a shallow model with applications to the wind driven circulation," Nonlinear Proc. Geophys. 2, 241 (1995).

${ }^{12}$ H. A. Dijkstra and C. A. Katsman, "Temporal variability of the winddriven quasi-geostrophic double gyre ocean circulation: Basic bifurcation diagrams," Geophys. Astrophys. Fluid Dyn. 85, 195 (1997).

${ }^{13}$ C. K. Mamum and L. S. Tuckerman, "Asymmetry and hopf bifurcation in spherical couette flow," Phys. Fluids 7, 80 (1995).

${ }^{14}$ M. D. Graham, U. Muller, and P. H. Steen, "Time-periodic thermal convection in Hele-Shaw slots: The diagonal oscillation," Phys. Fluids A 4, 2382 (1992).

${ }^{15}$ P. Holmes, J. L. Lumley, and G. Berkooz, Turbulence, Coherent Structures, Dynamical Systems and Symmetry (Cambridge University Press, Cambridge, UK, 1996).
${ }^{16}$ A. C. Newell and J. A. Whitehead, "Finite bandwidth, finite amplitude convection," J. Fluid Mech. 38, 279 (1969).

${ }^{17}$ M. Renardy and Y. Renardy, "Derivation of amplitude equations and analysis of sideband instabilities in two-layer flows," Phys. Fluids A 5, 2738 (1993).

${ }^{18}$ P. C. F. Van der Vaart and H. A. Dijkstra, "Sideband instabilities of mixed barotropic/baroclinic waves growing on a midlatitude zonal jet," Phys. Fluids 9, 615 (1997).

${ }^{19}$ E. N. Lorenz, "Deterministic nonperiodic flow," J. Atmos. Sci. 20, 130 (1963).

${ }^{20}$ J. H. Curry, J. R. Herring, J. Loncaric, and S. A. Orszag, "Order and disorder in two- and three dimensional Benard convection," J. Fluid Mech. 147, 1 (1984).

${ }^{21}$ L. Gardini, R. Lupini, and C. Tebaldi, "Bifurcations of steady forced flows in spectral models of rotating fluids," Phys. Fluids A 5, 2738 (1993).

${ }^{22}$ G. R. Flierl and K. Haines, "The decay of modons due to Rossby wave radiation," Phys. Fluids A 5, 2738 (1993).

${ }^{23}$ M. Golubitsky, I. Stewart, and D. G. Schaeffer, Singularities and Groups in Bifurcation Theory, Vol. II (Springer-Verlag, New York, 1988).

${ }^{24}$ H. B. Keller, in Applications of Bifurcation Theory, edited by P. H. Rabinowitz (Academic, New York, 1977).

${ }^{25}$ Y. Saad, Iterative Methods for Sparse Matrices (PWS, Boston, 1996).

${ }^{26}$ G. H. Golub and C. F. Van Loan, Matrix Computations (The Johns Hopkins University Press, Baltimore, MD, 1983).

${ }^{27}$ Y. A. Kuznetsov, Elements of Applied Bifurcation Theory (Springer Verlag, New York, 1995).

${ }^{28}$ E. Doedel and L. S. Tuckermann, Numerical Methods or Bifurcation Problems and Large-Scale Dynamical Systems (Springer, New York, 2000).

${ }^{29}$ E. Simonnet and H. A. Dijkstra, "Spontaneous generation of lowfrequency modes of variability in the wind-driven ocean circulation," J. Phys. Oceanogr. 32, 1747 (2002).

${ }^{30}$ R. Seydel, Practical Bifurcation and Stability Analysis: From Equilibrium to Chaos (Springer-Verlag, New York, 1994).

${ }^{31}$ H. A. Dijkstra, Nonlinear Physical Oceanography (Kluwer Academic, Dordrecht, the Netherlands, 2000).

${ }^{32}$ G. L. G. Sleijpen and H. A. Van der Vorst, "A Jacobi-Davidson iteration method for linear eigenvalue problems," SIAM J. Matrix Anal. Appl. 17, 410 (1996). 